\title{
Molecular-dynamics simulations of interfaces between water and crystalline urea
}

\author{
E. S. Boek, W. J. Briels, J. van Eerden, and D. Feil \\ Chemical Physics Laboratory, University of Twente, P.O. Box 217, 7500 AE Enschede, The Netherlands
}

(Received 8 October 1991; accepted 29 January 1992)

\begin{abstract}
Molecular-dynamics simulations of several water-crystalline urea interfaces have been performed. The structure and dynamics of water close to the urea crystal surface are discussed in terms of density profiles, positional and orientational distribution functions, and diffusion coefficients. The water structure close to the interface is strongly determined by the structure of the crystal surface: the (001) and (111) interfaces reveal strong adsorption of water while

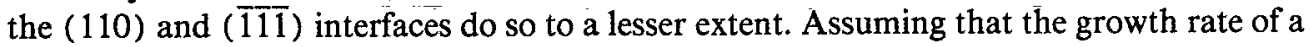
specific crystal face decreases with increasing solvent adsorption, the appearance of only (111) on the urea growth form is predicted. We argue that on the other hand the dominance of (110) over (001) cannot be explained using a simple layer growth model.
\end{abstract}

\section{INTRODUCTION}

The structure and dynamics of water molecules near crystalline surfaces play a fundamental role in crystal growth processes. Calculated habits derived from periodic bond chain (PBC) theory ${ }^{1}$ and Ising models ${ }^{2}$ generally are in good agreement with crystals grown by sublimation. When crystals are grown from solution, however, the resulting habit may deviate dramatically from calculated morphology. The reason is that the theoretical models consider only internal factors like crystal structure and bond energies; external factors such as impurities or crystal-solvent interaction are not taken into account. Nevertheless, these interactions are very important: It is generally accepted that preferential adsorption of solvent molecules on specific crystal faces leads to reduction of the growth rate of these faces. When the nature of these interactions is known and integrated in classical growth models, improved growth forms can be constructed. Berkovitch-Yellin ${ }^{3}$ was the first to approach this problem successfully in a semiquantitative way. Van der Voort ${ }^{4}$ proposed an expression for the growth rate including crystal-solvent interaction energies. However, both authors use only energies from static models. In the last decade a number of molecular-dynamics simulations have shown that water near a solid surface displays ordered layers. ${ }^{5-7}$ In this paper the dynamics and structure of water close to several urea surfaces are investigated. We shall shortly show why a study like this is realistic. Let us first mention the reasons for choosing this system.

(i) From aqueous solution urea crystallizes as long needles with large $\{110\}$ and small $\{001\}$ faces (length:breadth ratio of 50:1) ${ }^{8}$ Urea crystals from the vapor show the same faces but are more equidimensional (ratio of $4: 1$ ),${ }^{9}$ which is in good agreement with theory. ${ }^{10}$ We want to investigate whether water adsorption is strongest on $\{110\}$, causing retardation of the growth of this face.

(ii) A famous crystallographic problem is why only one of the faces (111) and ( $\overline{11 \overline{1}})$ appears on urea crystals and the other one is never observed. PBC theory does not discriminate between these two faces since the slice energies (energy released per molecule when a new growth slice is formed from the vapor) are the same. Specific adsorption of water molecules may be an explanation.

(iii) For both urea and water, potentials are available.

In view of the high solubility of urea in water it might be suspected that an analysis of interfaces between a flat urea crystal and pure water is not very relevant to the study of "rapid" urea crystal growth from supersaturated aqueous solutions. Hereafter, we will give some arguments to justify this approximation. We first give the general reasoning, and then substantiate this by some numerical illustrations.

(1) Crystallization is a slow proccss compared to the Newtonian dynamics of water and urea. We want to emphasize that we do not study the crystallization process itself, but investigate the parameters which are important to this. These parameters have to be calculated as averages over the fast (Newtonian) variables.

(2) A comparable argument applies to the spatial scales. As the urea crystal surfaces under consideration are connected nets, ${ }^{10}$ they consequently grow according to a layer mechanism ${ }^{1}$ and will certainly be flat on a microscopic scale.

(3) Is it realistic to assume pure water above the urea crystal? The rate-determining step for crystal growth from solution is the adsorption rate of urea. This rate is determined by two quantities: First, by the number of urea molecules colliding with the surface and the corresponding frequency; and second, by the adsorption probability per collision. The last quantity is determined by the answer to the question: What does the urea molecule see when it collides with the surface? $\Lambda$ s the adsorption-desorption rate for water is much higher than for urea, the urea molecule sees a crystal surface with water molecules adsorbed to it in dynamical equilibrium. In the following we will show that the distribution of the adsorbed water molecules is mainly determined by the crystal surface structure. Therefore we think that the presence of urea in the fluid will influence the water distribution only slightly.

We now want to illustrate these three arguments numerically. 
(ad 1) Experimental evidence for the fact that crystallization takes place at a much larger time scale than a typical simulation time of some tens of picoseconds is given by measured urea growth rates on the order of $10^{-6} \mathrm{~m} / \mathrm{s}(\approx 1 \mathrm{mo}-$ lecular growth layer per $\left.10^{-4} \mathrm{~s}\right){ }^{8}$ Therefore it can safely be stated that no perceptible growth would occur during the considered simulation time.

(ad 1,3) Test $N p T$ simulations of a dynamic urea crystal with pure water (allowing urea solvation) do not show solvation of any urea moleculc during a simulation time of 50 ps. Besides, the motion of the surface molecules will mainly be described by the excitations of a few slow surface phonons of long wavelength. This justifies the approximation of keeping the crystal rigid during the simulation runs.

(ad 1,3) Experimental diffusion coefficients of urea in moderately concentrated aqueous solutions are on the order of $10^{-5} \mathrm{~cm}^{2} / \mathrm{s}^{11}$ Urea molecules diffuse over $1000 \mathrm{~nm}$ during the time the crystal grows $1 \mathrm{~nm}$, i.e., the adsorption-desorption frequency is rate determining. For water this is on the order of $10^{12}$ molecules $\mathrm{s}^{-1} \mathrm{~nm}^{-2}$ (this study) and for urea $10^{4}$ molecules $\mathrm{s}^{-1} \mathrm{~nm}^{-2}$. $^{8}$

(ad 2) Test $N p T$ simulations of a dynamic urea crystal with pure water do not even show the beginning of surface reconstruction. To really detect surface reconstruction, this method would probably require prohibitively long calculation times.

\section{DETAILS OF THE SIMULATION}

Molecular-dynamics simulations have been performed using the GROMOS (Ref. 12) package. Four surfaces are investigated: (001), (110), (111), and (111). The central computational box consists of a urea crystal and 570 water molecules. The crystal is constructed by cutting the desired surface out of a bulk crystal which is rotated so that the surface under consideration is perpendicular to the $z$ axis. Then the box is filled up with water molecules in the positive $z$ direction. Thus structural effects can be described as a function of the coordinate $z$ (distance from the surface). Periodic boundary conditions are applied in three dimensions. In the $x$ and $y$ directions periodicity is preserved by choosing the side faces of the crystal to be planes with integer indexes. For (001) and (110) it was possible to construct a rectangular crystal box, while for (111) a monoclinic box with $\beta=80.7^{\circ}$ was generated. In the $z$ direction a sequence of water layers and crystal layers is obtained. Due to crystal symmetry the crystal exposes both of the two symmetric opposite surfaces to the water molecules. This means that the coupled faces $(001)$ and $(00 \overline{1})$ can be simulated in one run. The same holds for the couple (110) and ( $\overline{110})$ and for the polar couple (111) and (i11). For details about the computational boxes we refer to Table I.

For water the SPC model ${ }^{13}$ was used $\left(\rho=1.0 \mathrm{~g} / \mathrm{cm}^{3}\right)$, while the cell constants and atom coordinates for urea were taken from Hagler, Huler, and Lifson (HHL) ${ }^{14}$ Different urea force fields were tested by performing an isothermal isobaric, i.e., constant $-N p T$ simulation $(T=293 \mathrm{~K}, p=1$ atm) of the urea crystal ( $4 \times 4 \times 5$ unit cells) without water. The GROMOS, ${ }^{15}$ AMBER, ${ }^{16}$ (without hydrogen-bond potential), and OPLS (Ref. 17) potentials yielded a large vol-
TABLE I. Dimensions ${ }^{a}$ of the urea crystal boxes in $x, y$, and $z$ directions, thickness of the water layer in the $z$ direction before equilibration $(\mathrm{nm})$, and number of urea molecules in the box.

\begin{tabular}{|c|c|c|c|}
\hline & $(001)$ & (110) & (111) \\
\hline$x$ & $4 d_{100}=2.2644$ & $5 d_{001}=2.3560$ & $8 d_{11 \overline{1}}=2.4731$ \\
\hline$y$ & $4 d_{010}=2.2644$ & $6 d_{\overline{110}}=2.4018$ & $6 d_{\overline{1}_{10}}=2.4018$ \\
\hline$z$ & $5 d_{\infty O 1}=2.3560$ & $10 d_{110}=4.0030$ & $7 d_{11 \mathrm{t}}=2.1357$ \\
\hline$z_{\text {water }}$ & 3.4693 & 3.2120 & 3.0743 \\
\hline$n_{\text {urea }}$ & 160 & 300 & 168 \\
\hline
\end{tabular}

a $d_{h k l}$ is defined as the distance between two lattice planes $(h k l)$.

ume decrease of respectively $14 \%, 15 \%$, and $31 \%$ with respect to the experimental volume. The HHL ${ }^{14}$ potential (supplemented with force constants from GROMOS) displayed a 5\% volume increase. Bond lengths and anisotropic thermal parameters were calculated from average coordinates and rms fluctuations of atom coordinates, respectively. The GROMOS, AMBER, and OPLS force fields show a bad agreement with neutron-diffraction data. ${ }^{18}$ The HHL potential yields satisfactory bond lengths (conidering thermal shortening) but the thermal parameters are much too large (Table II). In order to improve this quantity we plan to introduce Ewald summations in the GROMOS package as these are not available at the moment. A test $N p T$ simulation run of a urea crystal with water shows thermal parameters in the urea surface layers which are even larger. It will be clear that this too large thermal motion will influence the water structure close to the interface in an undesirable way. Therefore it was decided to perform $N V T$ simulations with the HIL potential, keeping the positions of the atoms in the urea crystal fixed. In this way we can focus entirely on the water structure and dynamics, although we realize that a slight bias might be introduced by completely neglecting the crystal dynamics. The justification for this simplification has

TABLE II. Comparison of bond lengths and thermal parameters of urea at $293 \mathrm{~K}$ and $1 \mathrm{~atm}$, from $N p T$ molecular-dynamics (MD) and neutron-diffraction experiments (Ref. 18) (Expt.).

\begin{tabular}{|c|c|c|c|c|c|}
\hline & & \multicolumn{4}{|c|}{ Length $(\AA)$} \\
\hline \multicolumn{2}{|c|}{ Bond } & \multicolumn{2}{|r|}{ Expt. } & \multicolumn{2}{|c|}{ MD } \\
\hline \multicolumn{2}{|c|}{$\mathrm{C}-\mathrm{O}$} & \multicolumn{2}{|r|}{1.246} & \multicolumn{2}{|c|}{1.211} \\
\hline \multicolumn{2}{|c|}{$\mathbf{C}-\mathbf{N}$} & \multicolumn{2}{|r|}{1.333} & \multicolumn{2}{|c|}{1.302} \\
\hline \multicolumn{2}{|c|}{$\mathrm{N}-\mathrm{H}_{1}$} & \multicolumn{2}{|r|}{1.004} & \multicolumn{2}{|c|}{0.976} \\
\hline \multicolumn{2}{|c|}{$\mathbf{N}-\mathrm{H}_{2}$} & \multicolumn{2}{|r|}{1.005} & \multicolumn{2}{|c|}{0.982} \\
\hline \multicolumn{2}{|c|}{$\mathrm{N}-\mathrm{H}_{1} \cdots \mathrm{O}$} & \multicolumn{2}{|r|}{3.058} & \multicolumn{2}{|c|}{3.040} \\
\hline \multicolumn{2}{|c|}{$\mathrm{N}-\mathrm{H}_{2} \cdots \mathrm{O}$} & \multicolumn{2}{|r|}{3.081} & \multicolumn{2}{|c|}{2.977} \\
\hline \multicolumn{6}{|c|}{ Thermal parameters $U_{u l}$ (in units of $10^{-4} \AA^{2}$ ) } \\
\hline \multicolumn{3}{|c|}{$U_{11}$} & \multicolumn{3}{|c|}{$U_{33}$} \\
\hline Atom & Expt. & MD & Atom & Expt. & $\mathrm{MD}$ \\
\hline $\mathrm{C}$ & 353 & 617 & $\mathrm{C}$ & 155 & 258 \\
\hline 0 & 506 & 951 & 0 & 160 & 259 \\
\hline $\mathbf{N}$ & 692 & 809 & $\mathbf{N}$ & 251 & 401 \\
\hline $\mathrm{H}_{1}$ & 838 & 1201 & $\mathbf{H}_{1}$ & 478 & 725 \\
\hline $\mathbf{H}_{2}$ & 853 & 1056 & $\mathrm{H}_{2}$ & 267 & 390 \\
\hline
\end{tabular}


been given in the Introduction. The different crystal and water layers have a minimum thickness of about 20 and $30 \AA$ in the $z$ direction, respectively. This is acceptable in view of a cut-off distance for nonbonded interactions of $8.5 \AA$ : thus water molecules at different sides of the crystal cannot interact.

After the computational boxes were generated, energy minimizations were performed to remove spurious strain. Velocities from a Maxwellian distribution were assigned to the water molecules. The temperature of the water was maintained at $298 \mathrm{~K}$ by scaling the velocities with a time constant $\tau_{T}$ of $0.1 \mathrm{ps} .{ }^{19}$ Covalent bonds were constrained with SHAKE, allowing an integration time step of $2 \mathrm{fs}$. The system was allowed to equilibrate during a simulation run of $20 \mathrm{ps}$, followed by a production run of $30 \mathrm{ps}$. Quantities like energies, pressure, and temperature were stored every 0.02 ps, atomic coordinates and velocitics cvery 0.05 ps.

\section{STRUCURE AND DYNAMICS}

\section{A. Density profiles}

The density profiles of the centers of mass of the water molecules perpendicular to the surface are shown in Fig. 1. In the case of (001) and (110), the simulation boxes are symmetric along the $z$ direction, as might be expected on account of crystal symmetry and periodic boundary conditions. Due to the hemihedral symmetry of the urea crystal the surface structures of (111) and (i11) differ from each other. Consequently, the density profile near (111) is different from the profile near ( $\overline{111})$. Both (001) and (111) show two well-separated peaks close to the surface. For (110) and (111) two weakly pronounced water layers are observed. Only (001) displays a small third peak as well. From the minima in these density plots boundaries for the first two water layers are defined which are given in Table III, together with the centers of mass of the crystal layers next to the water. The liquid density in the center of the water box is somewhat lower than the SPC bulk water density of $1 \mathrm{~g} / \mathrm{cm}^{3}$ due to fact that the water effectively "wets" the crystal faces. It was shown ${ }^{6}$ that the height and positions of the density oscillations are quite insensitive to the precise value of the density in the center of the box. Therefore the density peaks can safely be described as adsorption of water layers.

\section{B. Scatter plots}

We also have investigated how the water molecules within these layers are distributed in the horizontal $x y$ plane. For this purpose scatter plots have been constructed (Fig. 2 ). In these figures dots are drawn at regular time intervals for every center of mass of a water molecule present in the layer. In Table III it is shown how many water molecules are present in these layers averaged over 30 ps of simulation time, from which also the average densities of the layers are calculated. For layer 1 these have to be regarded with care: only a part of the layer can be occupied with water, the remainder of the volume is taken by urea molecules. From these scatter plots the structure of the water becomes more visible.

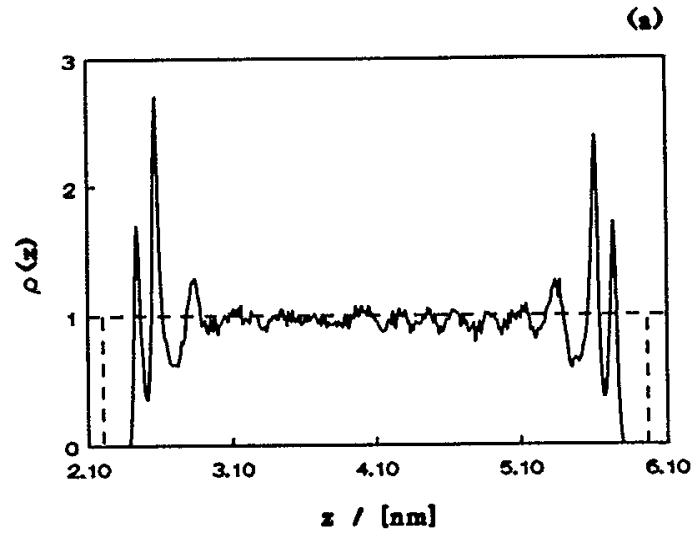

(b)

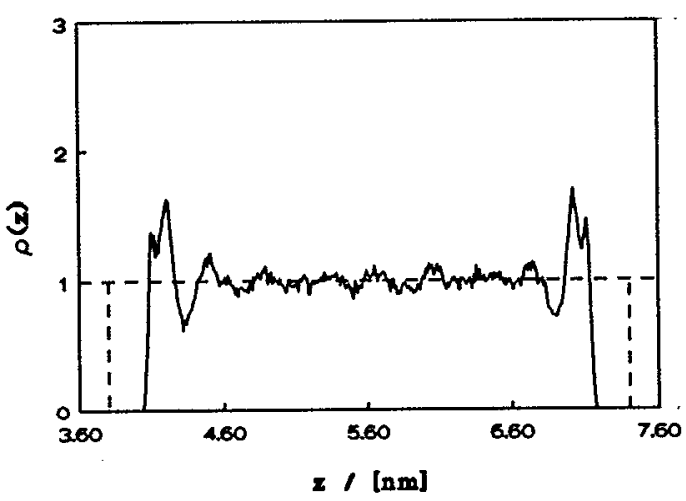

(c)

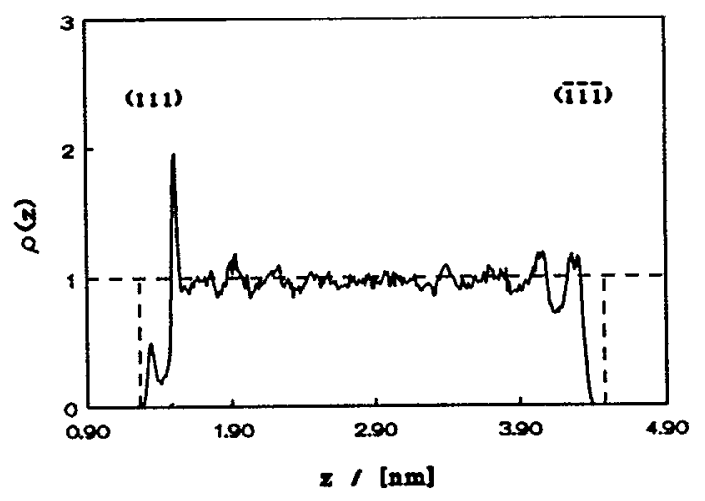

FIG. 1. Water density profiles at several urea surfaces. The centers of mass of the urea crystal boundary layers are designated by vertical dashed lines (Table III). The bulk water density is represented by a horizontal dashed line. (a) (001); (b) (110); (c) (111) and (111).

For the (001) surface the water in layer 1 is confined to trains of "pockets" in the surface along the diagonal [110] direction [Fig. 2(a1)]. When we look at a snapshot of this interface in the $x$ direction along the surface [Fig. 3(a)], we see water molecules pointing downward with one $\mathrm{OH}$ vector into such pockets in the surface. These pockets correspond to positions above the urea molecule $\mathbf{M ( 1 )}$ in a structure projection of urea along [001] in Fig. 4(a). The distance between the oxygen in $M(1)$ and the oxygen in water in layer 
TABLE III. Properties of adsorbed water layers. $z(u r)$ is the position of the center of mass of the crystal layer closest to the water, $z\left(m_{i}\right)$ is the position of the $i$ th minimum in the density profile [water layer 1, e.g., corresponds to $\left.z\left(m_{2}\right)-z\left(m_{1}\right)\right], n$-ads $(1)$ is the number of adsorption sites for layer $1, n-\mathrm{H}_{2} \mathrm{O}(i)$ is the number of water molecules present in layer $i$, and $\rho(i)$ is the density $\left(\mathrm{g} / \mathrm{cm}^{3}\right)$ of water layer $i$. The values of the last five quantities have been averaged for $(001)$ and $(00 \overline{1})$ and for $(110)$ and $(\overline{110})$, respectively.

\begin{tabular}{|c|c|c|c|c|c|c|}
\hline & $(001)$ & $(00 \overline{1})$ & (110) & (II0) & (111) & $(\overline{111})$ \\
\hline $\begin{array}{l}z(u r) \\
z\left(m_{1}\right)\end{array}$ & $\begin{array}{l}2.21 \\
2.38\end{array}$ & $\begin{array}{l}5.98 \\
5.80\end{array}$ & $\begin{array}{l}3.80 \\
4.03\end{array}$ & $\begin{array}{l}7.42 \\
710\end{array}$ & 1.27 & 4.48 \\
\hline$z\left(m_{2}\right)$ & $\begin{array}{l}2.50 \\
2.51\end{array}$ & 5.67 & 4.15 & 7.07 & $\begin{array}{l}1.21 \\
1.41\end{array}$ & $\begin{array}{l}4.40 \\
4.28\end{array}$ \\
\hline$z\left(m_{3}\right)$ & 2.68 & 5.50 & 4.32 & 6.90 & 1.55 & 4.14 \\
\hline$n-\operatorname{ads}(1)$ & \multicolumn{2}{|c|}{16} & \multicolumn{2}{|c|}{15} & 12 & 12 \\
\hline$n-\mathrm{H}_{2} \mathrm{O}(1)$ & \multicolumn{2}{|c|}{17} & \multicolumn{2}{|c|}{18} & 7 & 14 \\
\hline$n-\mathrm{H}_{2} \mathrm{O}(2)$ & \multicolumn{2}{|c|}{36} & \multicolumn{2}{|c|}{37} & 26 & 25 \\
\hline$\rho(1)$ & \multicolumn{2}{|c|}{0.77} & \multicolumn{2}{|c|}{0.80} & 0.25 & 0.59 \\
\hline$\rho(2)$ & \multicolumn{2}{|c|}{1.23} & \multicolumn{2}{|c|}{1.15} & 0.87 & 0.90 \\
\hline
\end{tabular}

1 shows a sharp preference for $0.3 \mathrm{~nm}$ which is typical for a hydrogen bond (Fig. 5). It can thus be said that the water molecules in layer 1 have a strong positional ordering imposed by the surface structure of the $(001)$ urea. Water layer 2, however, displays an almost bulklike structure [Fig. 2 (a2) ]. Only in the upper-right- and lower-left-hand parts of the unit cell a small increase of the density can be observed. From Figs. 3(a) and 4(a) it can be inferred that this is due to formation of hydrogen bonds to the $\mathrm{NH}_{2}$ groups of urea molecule $\mathbf{M}(2)$.

The first water layer at the (110) interface is quite diffuse compared to the one at the (001) surface [Fig. 2(b1)]. From a snapshot of this interface [Fig. 3(b)] it appears that the water molecules near the crystal point downward with one $\mathrm{OH}$ bond toward a flat-lying urea molecule which offers a lot of space to the water. Thus the water molecules can move rather easily along the $x$ axis. The $\mathrm{O}$ (urea) $-\mathrm{O}$ (water) distance distribution (Fig. 5) reveals again a maximum around $0.3 \mathrm{~nm}$ but is less sharply peaked than the one next to (001). From the scatter plots it can be seen that small water traces lead from one unit cell to the other along the $y$ axis. Apparently, the water can "jump" over the "wall" consisting of vertically oriented urea molecules [Fig. 3(b)] by forming hydrogen bonds to a $\mathrm{NH}_{2}$ group of this molecule [Fig. 4(b)], i.e., using the reversed Fosbury flop. Again, water layer 2 is much more diffuse and rather bulklike. A small density increase in the upper middle part of the unit cell points at hydrogen bonds with the vertical urea molecules.

Water layer 1 next to (111) is strictly confined to pockets in the surface offering hydrogen-bond possibilities to the oxygens of two urea molecules [see the snapshot in Figs. $3(c)$ and 4(c) ]. The surface offers 12 adsorption sites but these are occupied only half of the time as can be seen from Table II. This is in contrast with the first layers at the other faces, which display a complete occupation of the available adsorption sites. Layer 2 is ordered more strongly than the second layers of (001) and (110). The water is mainly bound to $\mathrm{NH}_{2}$ groups of the same urea molecules that bind the water molecules in layer 1 .
As can be seen from a snapshot along the ( $\overline{1} \overline{1})$ interface [Fig. 3(d)] water molecules in the first layer cannot penetrate into the pockets in the surface but are ordered along chains of urea molecules. The same holds for layer 2 to a lesser extent. Figures 3 (c) and 3(d) also reveal very clearly the difference between the (111) and (111) surface structures.

Generally, the positions of the water molecules in the two layers show a complementary pattern for the $(001)$, (110), and (111) surfaces while for ( $\overline{111})$ both layers show the same features. It is striking that both (111) and (i11) display a hexagonal pattern of adsorbed water molecules. Possibly, this accounts for the fact that the second water layers at these interfaces are strongly ordered compared to (001) and (110) due to the formation of an icelike structure.

\section{Orientational distributions}

We concluded from the scatter plots that the positional ordering of water is different for the four interfaces under investigation. From the snapshots in Fig. 3 there are indications that also the orientation of the water within these layers is different. In order to investigate this point the distribution of water dipole orientations has been calculated as a function of $\cos \theta$ [ $\theta$ is the angle between the dipole vector and the surface $x, y$, or $z$ (outward normal) axes], as well as the distribution of angles between the $\mathrm{OH}$ vectors and the surface outward normal vector (Fig. 6). These distributions have been determined for both water layers. For (001) and (110) the corresponding layers at both sides of the crystal have been averaged.

The first water layer adjacent to $(001)$ and (110) shows a random distribution for the angle between the dipole $x$ and $y$ components and the crystal $x$ and $y$ axes (not shown in a figure). The distribution of the $z$ components, however [Fig. 6(a) ], shows a strong preference for $\theta \sim 120^{\circ}$; from Fig. 6(c) it appears that water molecules in layer 1 point toward the surface with one $\mathrm{OH}$ vector around which the other $\mathrm{OH}$ vector can rotate freely. Thus the formation of a static hydrogen-bonded network with layer 2 is inhibited. This also 

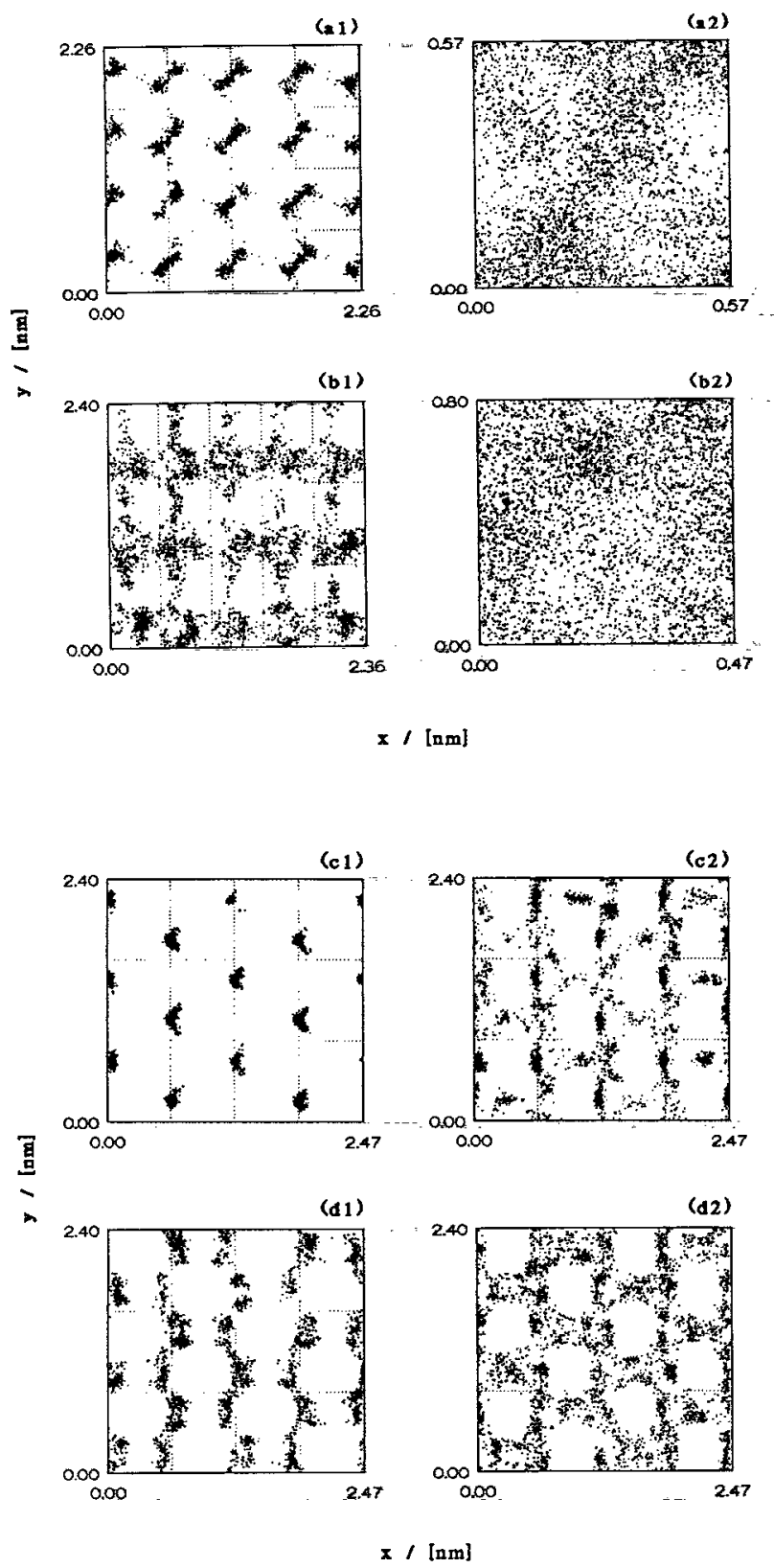

FIG. 2. Scatter plots of positions of water molecules on the $x y$ plane for both water layers near the various crystal surfaces. Layer boundaries are listed in Table III. Dots are drawn at regular time intervals varying for each layer so that \pm 4000 positions represent $30 \mathrm{ps}$ of simulation time. For the second layer near (001) and (110) the positions are transformed to the dimensions of one unit cell using crystal surface periodicity. (a1) layer 1 on ( $(01)$; (a2) layer 2 on (001); (b1) layer 1 on (110); (b2) layer 2 on (110); (c1) layer 1 on (111); (c2) layer 2 on (111); (d1) layer 1 on (111); (d2) layer 2 on (111).

explains why the second layer is so diffusely distributed in the $x y$ plane. The water in layer 2 shows a weakly pronounced preference for $\theta \sim 60^{\circ}$ for the dipole $z$ component [Fig. 6(b)] indicating hydrogen bridges with urea $\mathrm{NH}_{2}$ groups pointing out of the face and water molecules in layer 1. (a)

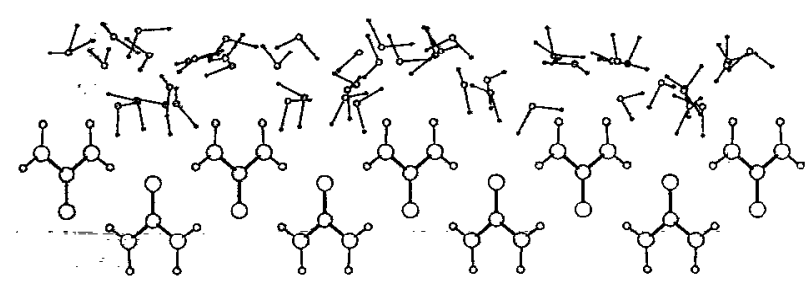

(b)

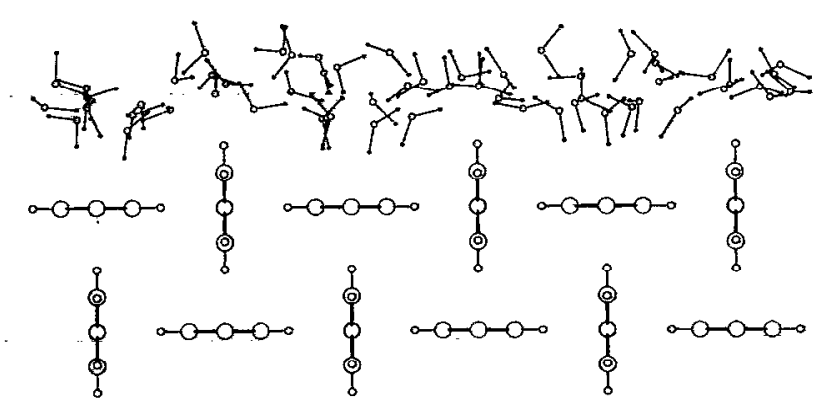<smiles>[Y][V]</smiles>

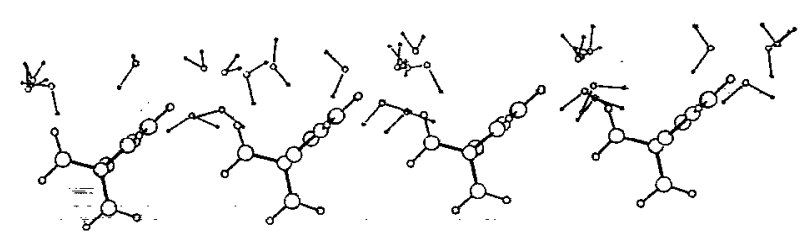

(d)

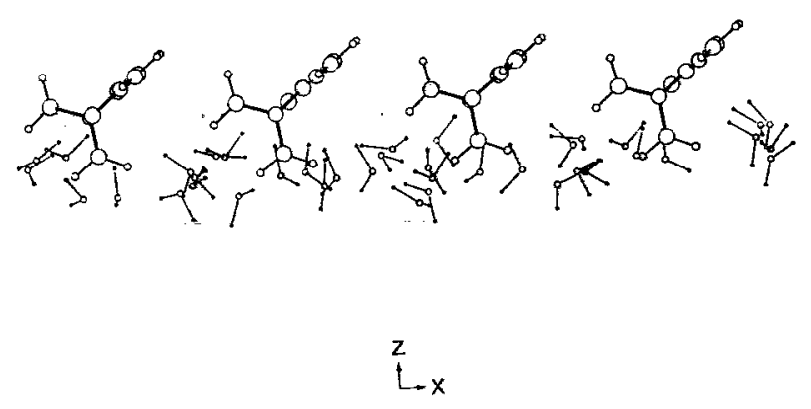

FIG. 3. Snapshots along the interfaces in the $x$ or $y$ direction (with respect to the urea crystal). Two urea boundary layers and all water molecules in two adsorption layers are drawn. (a) (001); (b) (110); (c) (111); (d) (i11). 
(a)

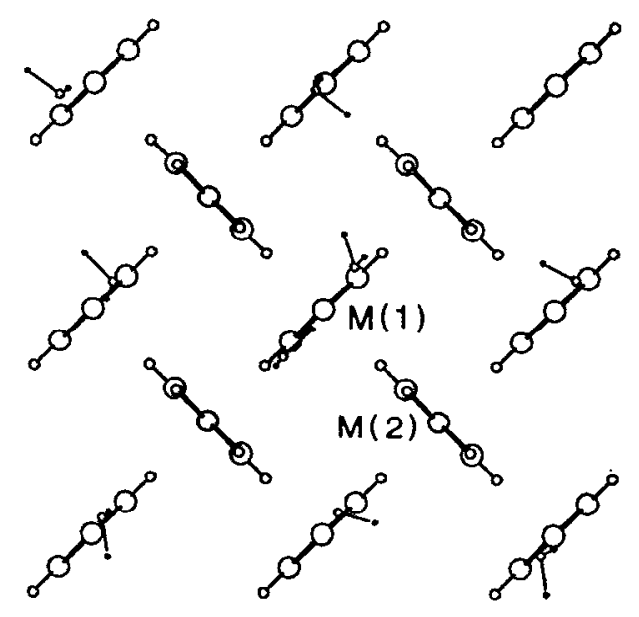

(b)

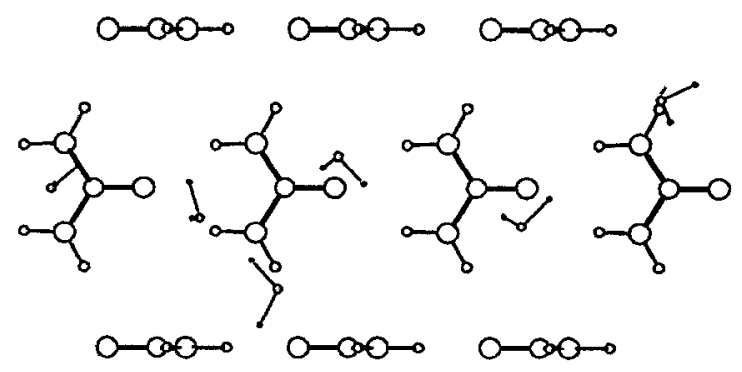<smiles>CC(C)OC(OC(C)C)C(C)O</smiles>

(c)

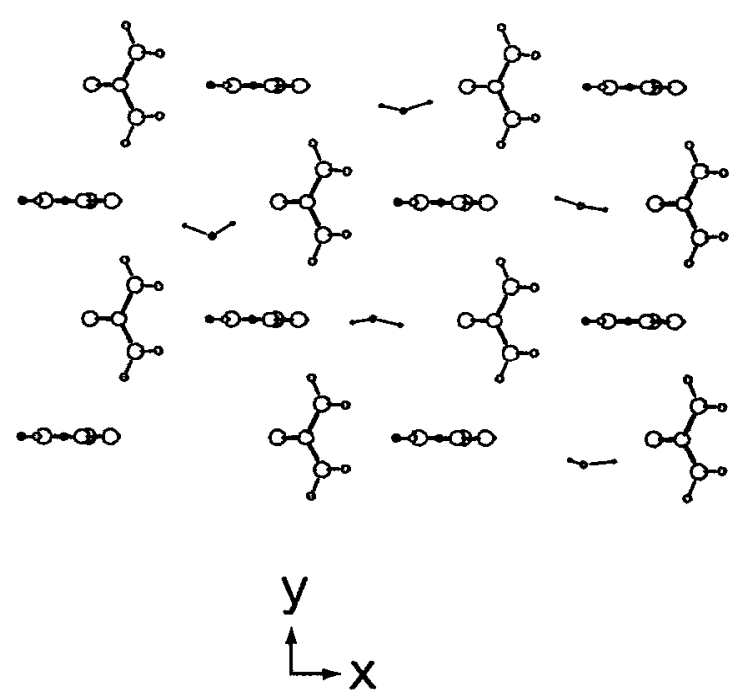

FIG. 4. Structure projections perpendicular to the interface. A part of the urea surface layer and the first adsorbed water layer are shown. (a) (001) ( $3 \times 3$ unit cells); (b) (110) ( $4 \times 2$ unit cells); (c) (111) ( $4 \times 2$ unit cells); The (III) projection is omitted as it follows directly from (c).

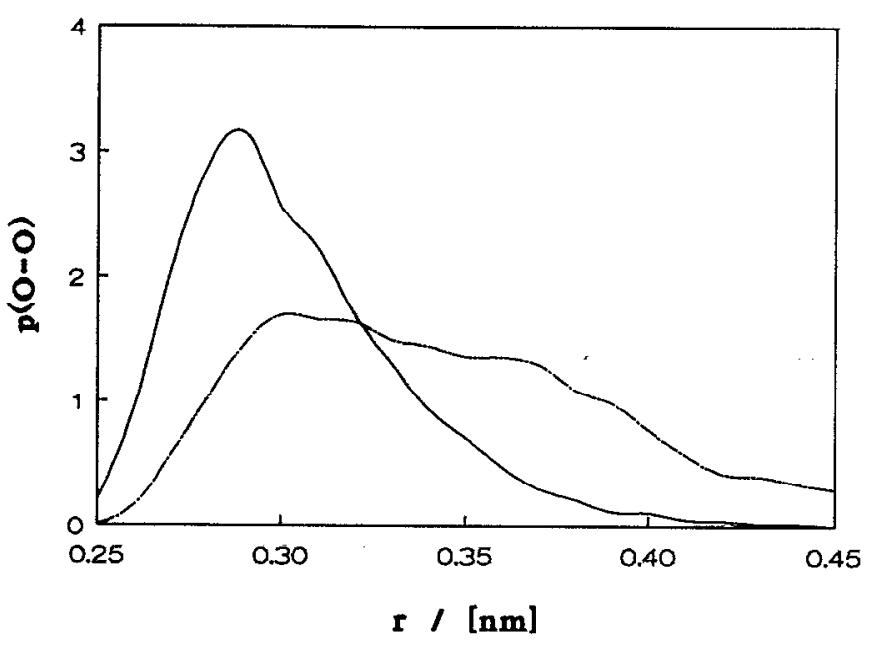

FIG. 5. Probability distribution of the distance between $O$ (urea) and $O$ (water) in the first water layer for the (001) (solid line) and (110) (dashdotted line) interfaces.

Layer 1 next to (111) shows a strong preference for $\theta \sim 180^{\circ}$ in the $z$ direction, so the water dipole points into the surface ["flop-down;" see also Fig. 3(c)]. The OH vectors show an enhanced probability for $\theta \sim 120^{\circ}$. Layer 2 prefers $\theta \sim 0^{\circ}$ for the dipole orientation ("flip-up"). No orientational ordering occurs for water next to ( $\overline{11} 1)$. The $\mathrm{OH}$ vectors in layer 2 show a random distribution for all interfaces (not shown).

\section{Diffusion}

The diffusional motion of the water molecules in a layer has becn calculated from the mean-square displacement, defined as

$$
\left\langle\Delta r^{2}(t)\right\rangle=\left\langle\left|\mathbf{r}\left(t_{0}+t\right)-\mathbf{r}\left(t_{0}\right)\right|^{2}\right\rangle
$$

in which we averaged over as many values of $t_{0}$ as possible and all centers of mass $i$. A correlation time of $10 \mathrm{ps}$ was taken. Effective diffusion coefficients were obtained from the Einstein relation,

$$
\begin{aligned}
& D_{x y}=\frac{1}{3} \lim _{t \rightarrow \infty} \frac{3}{2} \frac{\left\langle\Delta x(t)^{2}\right\rangle+\left\langle\Delta y(t)^{2}\right\rangle}{2 t}, \cdots \\
& D_{z}=\frac{1}{3} \lim _{t \rightarrow \infty} 3 \frac{\left\langle\Delta z(t)^{2}\right\rangle}{2 t},
\end{aligned}
$$

in which $\left\langle\Delta x(t)^{2}\right\rangle$ is the mean-square displacement in the $x$ direction. The diffusion coefficients for layers 1, 2, and bulk water near the different surfaces are shown in Table IV. The mean-square displacements for layer 1 in the $x y$ direction are shown in Fig. 7. Due to adsorption it may be expected that $D_{x y}$ (parallel to the the surface) is larger than $D_{z}$ (perpendicular to the surface) for water layers at all interfaces. Indeed, this appears to be true from Table IV. $D_{z}$ nearly equals zero for each surface. This result, however, should be regarded carefully due to the artificially imposed layer boundaries. The value of $D$ increases when going to the second layer and 
(a)

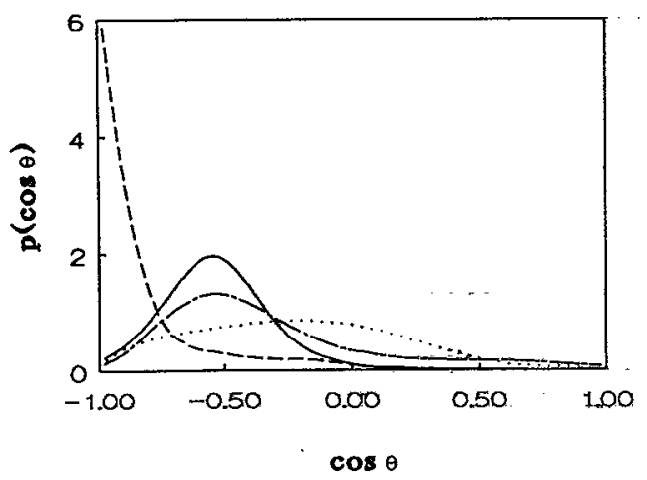

(b)

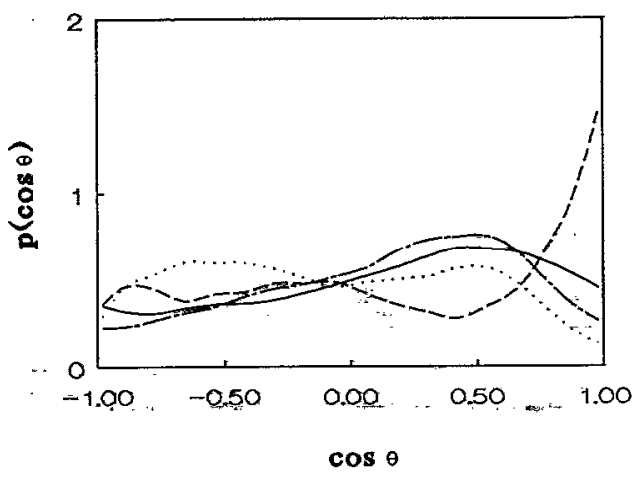

(c)

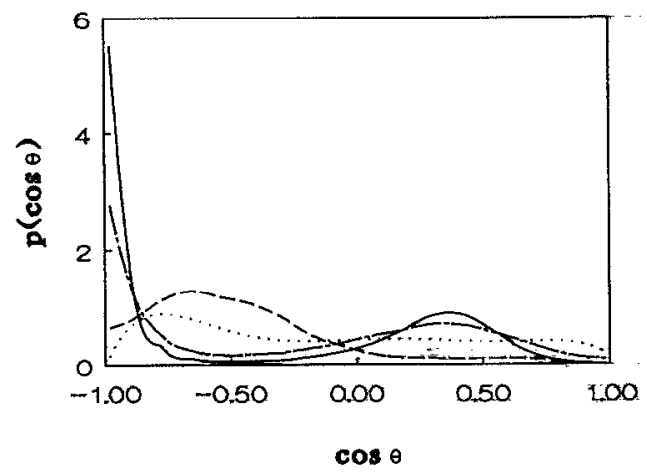

FIG. 6. Distribution of dipole and $\mathrm{OH}$ orientations as a function of $\cos \theta(\theta$ is the angle between the dipole or $\mathrm{OH}$ vector and the outward normal to the crystal surface) for (001) (solid line), (110) (dashed-dotted line), (111) (dashed linc), and ( $\overline{1} 1 \overline{1}$ ) (dotted line). (a) Dipole orientation for layer 1; (b) dipole orientation for layer 2 ; (c) $\mathrm{OH}$ orientation for layer 1.

the bulk which is in accordance with previous simulations. ${ }^{5,6}$ Even in bulk water the diffusion coefficient shows a slight anisotropy: $D_{z}$ remains a bit smaller than $D_{x y}$. Possibly, this is due to the fact that the water layer may not be thick enough to prevent interference bctween correlations due to the presence of the two surfaces.

The bulk diffusion coefficients compare quite well to

TABLE IV. Diffusion coefficients (in units of $10^{-5} \mathrm{~cm}^{2} / \mathrm{s}$ ).

\begin{tabular}{llllll}
\hline \hline Surface & Layer & $D_{x}$ & $D_{y}$ & $D_{x y}$ & $D_{z}$ \\
\hline (001) & 1 & 0.70 & 0.72 & 0.71 & 0.06 \\
& 2 & 1.62 & 1.52 & 1.57 & 0.14 \\
& bulk & 4.78 & 4.37 & 4.58 & 3.55 \\
$(110)$ & 1 & & & & \\
& 2 & 3.38 & 1.48 & 2.43 & 0.06 \\
& bulk & 2.90 & 2.33 & 2.61 & 0.19 \\
$(111)$ & 1 & 4.03 & 4.52 & 4.27 & 3.80 \\
& 2 & & & & \\
& bulk & 0.08 & 0.20 & 0.14 & 0.07 \\
$(\overline{111})$ & 1 & 4.48 & 0.79 & 0.66 & 0.07 \\
& 2 & 1.10 & 1.63 & 1.36 & 0.06 \\
& 1.45 & 1.35 & 1.40 & 0.14 \\
\hline \hline
\end{tabular}

results from previous simulations. ${ }^{6} D_{x}$ for (110) in layer 1 is much larger than $D_{y}$ which confirms our tentative picture of the water molecules moving easily in the $x$ direction (Sec. III B). Generally, it appears that there is no diffusion of

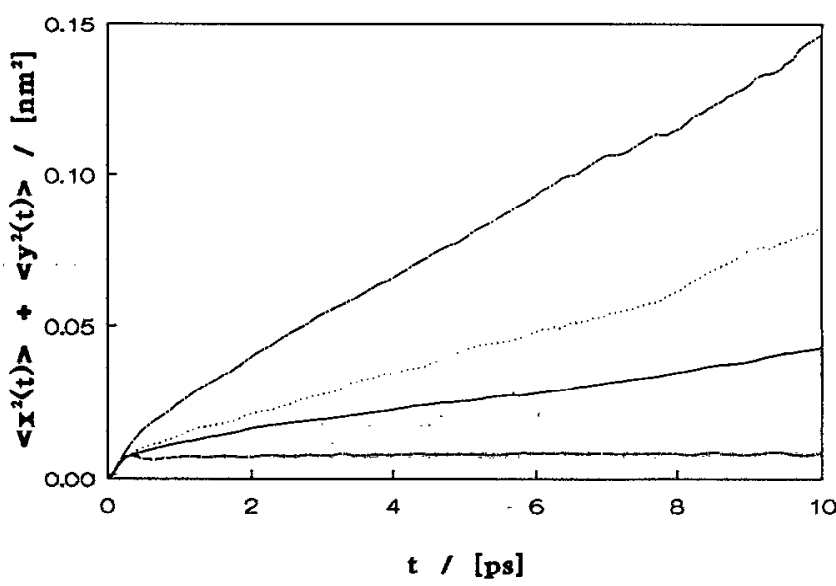

FIG. 7. Mean-square displacement in the $x y$ direction for layer 1 as a function of the correlation time for (111) (dashed line), (001) (solid line), (111) (dotted line), and (110) (dash-dotted line). 


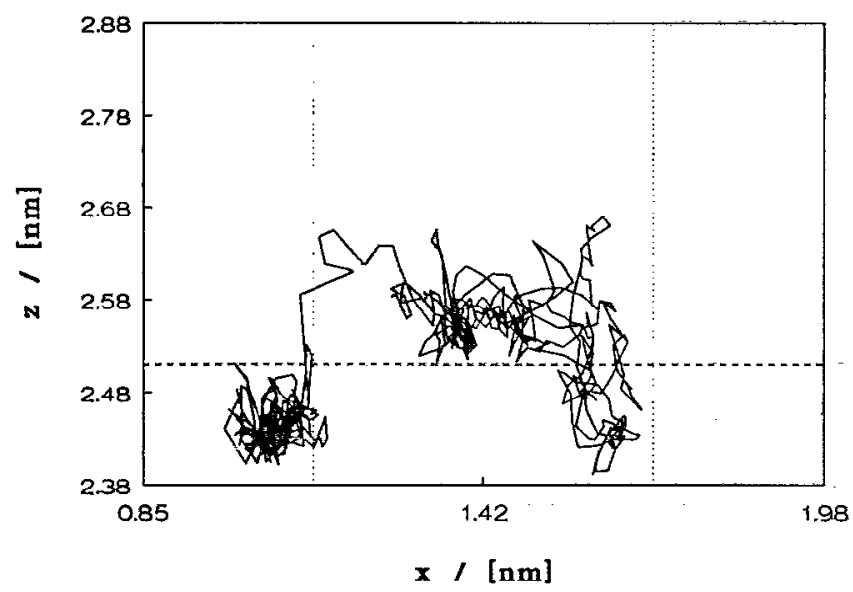

FIG. 8. Trajectory in the $x$ and $z$ directions for a water molecule hopping between two adsorption sites on (001). The motion in time is from left to right. The boundary between the first and second water layers is indicated by a horizontal dashed line; the vertical dotted lines represent urea unit-cell boundaries.

water parallel to (111). The diffusion increases when going from (001), ( $\overline{111})$ to (110). From the scatter plots of (001), however, it might be expected that no diffusion at all takes place within the first layer as there are no positions present between the adsorption sites. In the calculations water molecules were allowed to cross the layer boundaries as long as they returned within the simulation time of $30 \mathrm{ps}$. Therefore the diffusion in these layers should be entirely determined by water hopping from one adsorption site to the other via layer 2 or beyond. Indeed, several hopping water molecules have been observed by following their trajectories. One of them is drawn in Fig. 8 for the (001) interface.

\section{DISCUSSION AND CONCLUSIONS}

The structure and dynamics of water near crystalline urea surfaces have been investigated by means of moleculardynamics simulations. Density profiles perpendicular to the surface show two or three peaks with amplitudes strongly depending on the crystal surface. Both $(001)$ and (110) interfaces display only one adsorption layer with strong positional and orientational ordering while the second layer is liquidlike. Probably, the first water layer is "locked" into a position which is unfavorable for the formation of more ordered layers; ${ }^{20}$ the crystal forces water molecules in layer 1 into a position with one $\mathrm{OH}$ bond vector directed toward the surface around which the other $\mathrm{OH}$ bond can rotate freely. Thus the crystal surface is completely screened and water molecules in layer 2 "see" a flat hydrogen wall.

The (111) and ( $\overline{111}$ ) interfaces display different behavior: two ordered layers are observed, showing a hexagonal, icelike pattern. From scatter plots and density profiles we may infer that adsorption is stronger on (111) than on (111). This result is confirmed by the values of the coefficients for diffusion parallel to these interfaces. The consequences for urea crystal growth are important: strong ad- sorption on (111) compared to ( $\overline{11} 1)$ enhances the relative growth rate of $(\overline{11})$. Therefore we expect the appearance of only (111) on the growth form. Unfortunately, at present no data are available on the absolute polar morphology of the urea crystal to check this prediction.

From density profiles, positional order, and diffusion coefficients it appears that water adsorption is stronger on (001) than on (110). Consequently, we might expect that the growth of $(110)$ is enhanced relative to that of $(001)$. Experiments in aqueous solution, however, show that (110) has the larger surface area due to slower growth. We may think of several reasons for this contradiction. First, the relative water mobilities might change on simulating water restricted between dynamic crystal surfaces instead of static surfaces. We do not believe, however, that the dynamics of the crystal surface will do more than slightly smear the positional and time correlations in the adsorbed liquid film. Second, we should mention that it is not so much the mobilities of the water molecules that matter, but their desorption energies. Although there is some correlation between strong position correlation and small diffusion coefficients on the one hand, and adsorption energies on the other hand, this correlation is not $100 \%$. In order to formulate a third reason we return to the vacuum calculations of the urea crystal morphology. ${ }^{10}$ There it turned out that the slice energies and roughening temperatures of both faces are roughly equal, from which we concluded that they should have the same morphological importance. This conclusion was based on a layer-growth model in which growth occurs by means of diffusion of growth units to kink sites. Here we want to stress the role of the dynamics of the growth units which is not included explicitly in the classical layer-growth model: a newly formed growth layer will only then admit a next layer to be formed when it is completely rigid, i.e., solidified. From Fig. 3 it may be inferred that the mobility and therefore the entropy of adsorbed urea molecules is larger on the (110) face than on the (001) face. As a consequence the two-dimensional solidification temperature will be lower on the (110) face and its growth rate will be smaller. Stressing a slightly different aspect of the same argument we may say that for kink growth to occur, the kink must be perfectly rigid which is less probable on the (110) face than on the (001) face. In order to check this hypothesis we plan to perform simulations in order to calculate the free energies of removing solid urea molecules from different positions in different surfaces. Besides, simulations of interfaces between saturated urea solutions and the urea crystal are planned.

\section{ACKNOWLEDGMENTS}

We thank G. J. Hoevers for doing preliminary work and the use of his computer programs. The Netherlands Organization for Scientific Research (SON/NWO) is acknowledged for financial support.

\footnotetext{
${ }^{1}$ P. Hartman, in Crystal Growth: An Introduction, edited by P. Hartman (North-Holland, Amsterdam, 1973), p. 367.

${ }^{2}$ P. Bennema and J. P. van der Eerden, in Morphology of Crystals, edited by I. Sunagawa (TERRAPUB, Tokyo, 1987), p. 1.
} 
${ }^{3}$ Z. Berkovitch-Yellin, J. Am. Chem. Soc. 107, 8239 (1985).

${ }^{4}$ E. van der Voort, J. Cryst. Growth 110, 662 (1991).

${ }^{3}$ E. Spohr, J. Phys. Chem. 93, 6171 (1989).

${ }^{6} \mathrm{M}$. Schlenkrich, K. Nicklas, J. Brickmann, and P. Bopp, Ber. Bunsenges. Phys. Chem. 94, 133 (1990).

${ }^{7}$ K. Raghavan, K. Foster, K. Motakabbir, and M. Berkowitz, J. Chem. Phys. 94, 2110 (1991).

${ }^{8}$ R. Davey, W. Fila, and J. Garside, J. Cryst. Growth 79, 607 (1986).

${ }^{9}$ R. S. Feigelson, R. K. Route, and T.-M. Kao, J. Cryst. Growth 72, 585 (1985).

${ }^{10}$ E. S. Boek, D. Feil, W. J. Briels, and P. Bennema, J. Cryst. Growth 114, 389 (1991).

${ }^{11}$ L. S. Sorell and A. S. Myerson, AIChE J. 28, 772 (1982).

${ }^{12}$ W. F. van Gunsteren and H. J. C. Berendsen, Groningen Molecular Simulation Library, 1987.
${ }^{13}$ H. J. C. Berendsen, J. P. M. Postma, W. F. van Gunsteren, and J. Hermans, in Intermolecular Forces, edited by B. Pullman (Reidel, Dordrecht, 1981), p. 331.

${ }^{14}$ A. T. Hagler, E. Huler, and S. Lifson, J. Am. Chem. Soc. 96, 5319 (1976).

is J. Hermans, H. J. C. Berendsen, W. F. van Gunsteren, and J. P. M. Postma, Biopolymers 23, 1513 (1984).

${ }^{16}$ S. J. Weiner, P. A. Kollman, D. T. Nguyen, and D. A. Case, J. Comput. Chem. 7, 230 (1986).

${ }^{17}$ W. L. Jorgenson and C. J. Swenson, J. Am. Chem. Soc. 107, 569 (1985).

${ }^{18}$ H. Gulh, G. Heger, S. Klein, W. Treutmann, and S. Scheringer, Z. Kristallogr. 153, 237 (1980).

${ }^{19}$ H. J. C. Berendsen, J. P. M. Postma, W. F. van Gunsteren, A. Di Nola, and J. R. Haak, J. Chem. Phys. 81, 3684 (1984).

${ }^{20}$ H. R. Pruppacher and J. D. Klett, Microphysics of Clouds and Precipitation (Reidel, Dordrecht, 1978), p. 257. 